Methods High definition video recordings were collected from patients with non-dysplastic (ND-BE) and dysplastic (D-BE) BE undergoing endoscopy at UCLH. A protocol was used to record areas of interest after which a matched biopsy was taken to confirm the histological diagnosis. In a blinded manner, videos were shown to 3 expert endoscopists who interpreted them based on their $\mathrm{M}$ and $\mathrm{V}$ patterns, presence of nodularity, ulceration and suspected diagnosis. Acetic acid (ACA) was used in some cases. Data was inputted into the WEKA package to construct a decision tree for dysplasia prediction.

Results Videos from 47 patients (13 before and after ACA) were collected (24 ND-BE, 23 D-BE). Cases in which ACA was used, 7 had ND-BE and 6 D-BE. Experts' average accuracy for dysplasia prediction was $72.2 \%$ (66.7-76.7\%). ACA did not improve dysplasia detection. In 5 cases all 3 experts failed to detect D-BE.

Using ML, the most important attribute was the lesions' $\mathrm{V}$ pattern. If this was reported abnormal (irregular, dilated vessels) by more than one doctor, the lesion was D-BE (accuracy 79\%). If $\mathrm{D}-\mathrm{BE}$ was predicted despite the $\mathrm{V}$ pattern being reported abnormal by one or fewer experts, the lesion was still D-BE and vice versa.

Conclusion Experts can diagnose D-BE in up to three-quarters of cases using i-Scan. ML can define rules learnt from expert opinion that predict dysplasia with a similar level of accuracy and are easier to learn than conventional classification systems. They could be used to train non-expert endoscopists in dysplasia detection and then used for blinded assessment of accuracy. Disclosure of Interest None Declared.

\section{PTU-059 COMPARISON OF BIPOLAR RADIOFREQUENCY CUTTING AND MONO POLAR CUTTING FOR ENDOSCOPIC SUBMUCOSAL DISSECTION (ESD) IN A PORCINE MODEL}

${ }^{1}$ ZP Tsiamoulos*, ${ }^{2} \mathrm{C}$ Hancock, ${ }^{3} \mathrm{PD}$ Sibbons, 'BP Saunders. 'Wolfson Unit for Endoscopy, St Mark's Hospital/Academic Institute, London, UK; ${ }^{2}$ Department of Electronic Engineering, Bangor University, Bangor; ${ }^{3}$ Department of Surgical Sciences, Northwick Park Institute for Medical Research, London, UK

10.1136/gutjnl-2014-307263.133

Introduction Current endoscopic knives utilise mono-polar energy to incise the mucosa, dissect the submucosa and coagulate bleeding vessels. Monopolar devices have proven efficacy but remain technically challenging to use with the risk of major complications.

Methods A new bipolar endoscopic device "Speedboat-RS2, (S-RS2) Creo Medical Ltd, UK" was compared to a standard mono-polar endoscopic device (Flush-knife-BT/F-BT/Fujifilm, Japan) for endoscopic submucosal dissection (ESD) in the porcine colon. The S-RS2 blade delivers bipolar radio frequency (RF-400 KHz) cutting and microwave coagulation $(5.8 \mathrm{GHz})$ for hemostasis, and contains a retractable needle for submucosal injection/tissue irrigation. It also has an insulated hull to prevent thermal injury to the underlying muscle layer. ESD was performed in a random order and video recorded on 5 consecutive $60 \mathrm{~kg}$ pigs. The following parameters were measured: time taken to complete resection, complications encountered and histological assessment. Two animals were recovered for one week and four animals for four weeks.

Results Ten consecutive resections were performed in the colorectum ( 2 per animal), 5 with S-RS2 and 5 with F-BT. Median time for S-RS2 to complete a resection was 44 min using RF cutting $30 \mathrm{~W}$, and for F-BT was $52 \mathrm{~min}$ using monopolar cutting for mucosal incision $(80 \mathrm{~W})$ and for submucosal dissection, monopolar forced coagulation 30W. Median flap size for S-RS2 was $36.8 \mathrm{~mm}$ and for F-BT was $43 \mathrm{~mm}$. Microwave coagulation was applied for either minor bleeding or visible vessels on 25 occasions with S-RS2. Monopolar coagulation was applied 14 times with F-BT, mean energy 30W. The Hemostatic Coagrasper was used 7 times to control arteriolar bleeding during S-RS2 dissection when microwave was not sufficient and only once during Flushknife-RS2 dissection. Endoclips were placed to treat deep muscle injury in the resection base on 10 occasions in the F-BT resections (15clips placed) and on 3 occasions (3 clips) for the S-RS2 resections. There was only one study perforation - F-BT group, where urgent peritoneal decompression was required and the resection was abandoned. Histology (S-RS2 resections) showed an intact muscle layer in four resection bases and in one there was slight muscle alteration but muscle cell viability was retained. The muscle layer was absent in two F-BT resection bases and moderately altered in one.

Conclusion Compared to Flush knife-BT ESD colonic resections (monopolar) the Speedboat-RS2 was was associated with less muscle injury and need for endoscopic clipping. However Speedboat-RS2 resections produced more intraprocedural bleeding requiring the haemostatic forceps.

Disclosure of Interest Z. Tsiamoulos Consultant for: Creo Medical Ltd, C. Hancock Shareholder of: Creo Medical Ltd, P. Sibbons Paid instructor for: Creo Medical Ltd, B. Saunders Consultant for: Creo Medical Ltd, Paid instructor for: Olympus KeyMed.

\section{Inflammatory bowel disease I}

\section{PTU-060 VACCINATING PATIENTS WITH IBD-STILL TO BEGIN, AT THE BEGINNING...}

${ }^{1} \mathrm{~A}$ Goel ${ }^{*},{ }^{2} \mathrm{CJ}$ Hill, ${ }^{2} \mathrm{~T}$ Johnson, ${ }^{3} \mathrm{JK}$ Limdi. ${ }^{1}$ Gastroenterology, Blackpool Teaching Hospitals NHS Trust, UK; ${ }^{2}$ Fylde and Wyre CCG, Blackpool, UK; ${ }^{3}$ Gastroenterology, Pennine Acute Hospitals NHS Trust, Bury, UK

\subsection{6/gutjpl-2014-307263.134}

Introduction Evolving definitions of disease control over the last decade have translated into earlier and often combined use of immunomodulatory (IM) therapy with the aim of achieving deep

Abstract PTU-060 Table 1 Data on Vaccinations administered to patients on immunomodulators

\begin{tabular}{|c|c|c|c|c|c|c|c|}
\hline & $\begin{array}{l}\text { Aza } \\
\text { (IBD patients) } \\
n=7\end{array}$ & $\begin{array}{l}\text { Aza } \\
\text { (non IBD patients) } \\
n=65\end{array}$ & $\begin{array}{l}\text { Metx } \\
\text { (IBD patients) } \\
n=31\end{array}$ & $\begin{array}{l}\text { Metx } \\
\text { (non IBD pts) } \\
n=415\end{array}$ & $\begin{array}{l}6 \mathrm{MP} \\
\text { (IBD pts) } \\
n=6\end{array}$ & $\begin{array}{l}\text { 6MP } \\
\text { (non IBD pts) } \\
n=7\end{array}$ & $\begin{array}{l}\text { Total patients } \\
\text { on IM } \\
n=594\end{array}$ \\
\hline Influenza vaccine. & $37(52.9 \%)$ & $49(75.4 \%)$ & $23(74.2 \%)$ & $317(76.4 \%)$ & $2(33.3 \%)$ & $2(28.6 \%)$ & $430(72.4 \%)$ \\
\hline Hepatitis B vaccine & $5(7.1 \%)$ & $18(27.7 \%)$ & $2(6.5 \%)$ & $21(5.1 \%)$ & 0 & 0 & $46(7.7 \%)$ \\
\hline HPV vaccine (females) & $2(6.8 \%)$ & $2(4.7 \%)$ & 0 & $4(1.3 \%)$ & $1(50 \%)$ & 0 & $9(2.3 \%)$ \\
\hline MMR vaccine & $16(22.9 \%)$ & $5(7.7 \%)$ & $1(3.2 \%)$ & $12(2.9 \%)$ & $1(16.7 \%)$ & $1(14.3 \%)$ & $36(6.1 \%)$ \\
\hline Pneumococcal vaccine & $28(40.0 \%)$ & $43(66.2)$ & $18(58.1 \%)$ & $259(62.4)$ & $2(16.7 \%)$ & $3(42.9 \%)$ & $353(59.4 \%)$ \\
\hline
\end{tabular}


remission. The potential for immunosuppression to increase the risk of opportunistic infection was recognised early with the publication of ECCO Consensus guidelines on prevention diagnosis and management of opportunistic infections in IBD. Has this permeated to the grass roots of clinical care or are we still "top-heavy"? We reviewed vaccination practices in a large primary care cohort.

Methods Data was obtained from primary care in the Fylde and Wyre CCG from electronic patient records maintained on EMIS Web. Patients were considered immunosuppressed if they were on doses of prednisolone $>20 \mathrm{mg} /$ day or equivalent for 2 weeks or more, ongoing treatment with effective doses of Thiopurines, Methotrexate, Infliximab and Adalimumab or had these agents discontinued within 3 months. We identified patients treated with these drugs between September 2012 and August 2013. Vaccination practices were reviewed in line with ECCO consensus. Data on biologic therapy and steroids was not available.

Results A total patient population of 93,240 (adult population of 75,952 ) from 13 practices was thus audited. A total of 594 patients were prescribed IM therapy [Azathioprine (AZA) 135, Methotrexate (MTX) 446, 6MP 13]. The data on vaccinations is shown in Table 1 . Vaccination rates were significantly higher in the non IBD cohort as compared to the IBD cohort, for influenza vaccine $\mathrm{p}<0.001,95 \% \mathrm{CI}(-0.17 \pm 0.1)$ and pneumococcal vaccine $\mathrm{P}<0.00195 \%$ CI $(-0.177 \pm 0.103)$.

Conclusion The practice of immunisation was poor and probably reflective of disparate practices elsewhere. Mere existence of guidelines is insufficient for quality improvement, which must take into account and remedy barriers to implementation. These include clinician awareness, concerns regarding safety and side effects and ambiguity regarding responsibility. Gastroenterologists must provide a clear line of communication to primary care physicians. We are taking necessary steps through a dedicated proforma to ensure vaccination uptake.

Disclosure of Interest None Declared.

\section{PTU-061 INFLIXIMAB OR CICLOSPORIN: PATIENTS' TREATMENT PREFERENCES AND THE IMPACT OF ULCERATIVE COLITIS (UC) ON THEIR LIVES}

A Seagrove ${ }^{*}$, F Rapport, J Williams. Swansea University, Swansea, UK

10.1136/gutjnl-2014-307263.135

Introduction The qualitative element of CONSTRUCT, a randomised controlled trial comparing clinical and cost effectiveness of infliximab and ciclosporin in steroid resistant UC, contributed to the specific trial objectives of examining quality of life (QoL) across the two groups. The qualitative element concentrated on patient and professional perceptions, and we describe patients' opinions about treatments and other UC therapies.

Methods Semi-structured telephone interviews with patients three and 12 months after admission with acute severe UC and randomisation. Thematic analysis was conducted by three qualitative researchers, followed by group analysis by seven members of CONSTRUCT. This abstract concentrates on the three month data.

Results 20 interviews were completed. Length of disease duration varied but similar stories emerged about living and coping with UC, the physical, mental and emotional impact of the disease, treatment options and concerns and hopes for the future. The main issues were:
- Patients favour infliximab because they perceive a more positive treatment outcome, easier treatment regime and fewer side effects

- The dramatically debilitating symptoms that impact on patients' QoL, their family and friends, are particularly noticeable in this disease

- Patients live with the ongoing unpredictability of symptoms and treatment, making it particularly difficult for patients and healthcare professionals to manage care

- Unlike other chronic diseases, UC is considered embarrassing, making it an isolating and awkward experience for patients and difficult to manage work and life routines

- The lack of visibility of symptoms or outcomes impacts on patients' ability to share and discuss openly with others

- Surgery is feared but most patients experience relief and subsequent recognition of health benefits following surgery

- Patients would like to understand what causes UC, its links to stressors and diet and would welcome more extensive information provision

- Ready access to IBD Nurses was considered important.

Conclusion Study findings indicate that UC patients live with constant, unpredictable symptoms, where a flare-up becomes socially isolating along with anxieties of deteriorating health. Patients need support to manage the impact of UC on their lives, consider prompt diagnosis to be important, and need relevant treatment provided quickly. Patients clearly prefer infliximab because of the easier treatment regime and fewer side effects. The views of patients after surgery were generally positive, but more research is required into surgical treatment of UC to support those facing surgery and as an alternative to medical treatment. The profile of UC should be raised to destigmatise the disease and thus the embarrassment felt by sufferers and those living with the outcomes of surgery.

Disclosure of Interest None Declared.

\section{PTU-062 CROHN'S DISEASE-ASSOCIATED ESCHERICHIA COLI CAN TOLERATE LOW NUTRIENT, LOW PH AND HIGH CHEMICAL STRESS ENVIRONMENTS ENCOUNTERED WITHIN THE PHAGOLYSOSOME OF MUCOSAL MACROPHAGES}

A Tawfik*, JM Rhodes, BJ Campbell. Gastroenterology, Institute of Translational Medicine, Liverpool, UK

\subsection{6/gutjnl-2014-307263.136}

Introduction Mucosa-associated adherent, invasive E.coli (AIEC), found in increased number in Crohn disease (CD) ileal and colonic mucosa, can replicate within host mucosal macrophages $^{1,2}$ Other pathogens, known to survive within macrophages (e.g. Mycobacteria and Salmonella), possess stressresponse inducible systems that allow them to tolerate environmental stress. Here we aimed to establish whether CD AIEC are better able than non-AIEC E.coli to tolerate low $\mathrm{pH}$, high nitrosative and oxidative stress conditions, that mimic the environment within the macrophage phagolysosome.

Methods CD AIEC isolates possessing ability to replicate intracellularly in J774-A1 macrophages (ileal isolate LF82 and 3 colonic isolates HM427, HM605 and HM615; range 4.5 to 9fold replication) were compared to E.coli strains killed by macrophages (XL1 and EPI300). Bacterial cultures were grown at $37^{\circ} \mathrm{C}$ in Luria-Bertani (LB) medium to $\mathrm{OD}_{600 \mathrm{~nm}} 0.1$ and diluted in 10 -fold steps in $0.9 \% \mathrm{w} / \mathrm{v}$ saline. Samples were spotted onto LB agar containing one of the following stress agents: $100 \mathrm{mM}$ 4-Morpholine ethanesulfonic acid (MES) pH5, 100mM MES 